\title{
Natural Heat Transfer Phenomenon in MHD Fractional Second Grade Fluid
}

\author{
Salah Uddin ${ }^{1, *}$, Mahathir Mohamad ${ }^{1}$, Mahmod Abd Hakim Mohmad ${ }^{2}$, Obaid Ullah Mehmood ${ }^{3}$, \\ MGhazali Kamardan ${ }^{1}$, Rozaini Roslan ${ }^{1}$ \\ ${ }^{1}$ Department of Mathematics and Statistics, Faculty of Applied Sciences and Technology, Universiti Tun Hussein Onn Malaysia, \\ ${ }^{2}$ Department of Mechanical Engineering, Faculty of Diploma Study Centre, Universiti Tun Hussein Onn Malaysia, Malaysia \\ ${ }^{3}$ Department of Mathematics, Comsats University Islamabad, Pakistan
}

Received July 30, 2019; Revised October 1, 2019; Accepted December 27, 2019

Copyright $@ 2019$ by authors, all rights reserved. Authors agree that this article remains permanently open access under the terms of the Creative Commons Attribution License 4.0 International License

\begin{abstract}
This paper aims at the heat transfer phenomenon and the effect of magnetic field on the second-grade fluid in a vertical oscillating cylinder. By applying a perpendicular magnetic field, the fluid gets magnetized. Fractional MHD flow was modeled with Caputo-Fabrizio non-integer derivative approach. Exact solution of the governing equations was obtained by Laplace and finite Hankel transforms. Mathematical computations and graphical plots were used to investigate the quantitative effects of emerging dimensionless physical parameters on the second-grade fluid flow, such as magnetic field and Prandtl number.
\end{abstract}

Keywords Magnetized Solution, Buoyancy Forces, Non-integer Derivative, Conventional Fluid, Fractional Fluid

\section{Introduction}

Nowadays BFD (biomagnetic fluid dynamics) and MHD (magneto hydrodynamics) are gaining significant attention in fractional-order electromagnetism, bio-engineering and neurons modeling in biology. Heat transfer has a major impact on the non-Newtonian flow problems in industry and engineering.

In the analytical study of Nehad and Ilyas [1], fractional parameter enhances the fluid velocity in the vertical oscillating plate. Das et al [2] applied Runge-Kutta sixth order method to the stretching heat model. The results conclude that thermal radiation significantly increases the boundary layer velocity and temperature. Rehman et al. [3] used a homotopy analysis method to find the Erying Powell fluid stagnation point inside the vertical cylinder. Keller box scheme was employed by Prasad et al. [4] to numerically simulate the incompressible second-grade fluid. Numerical results show that the heat transfer rate and velocity gradient decelerates with streamwise coordinate. Alao et al. [5] solved the viscous dissipation model by spectral relaxation method. It was shown that thermal radiation rise resulted in the cooling plate. Fourth-grade thin-film flow was analytically studied by using Adomian decomposition method and Homotopy asymptotic method by Gul et al. [6]. Graphical results were compared and found in good agreement with both of the methods. Visco-elastic fluid flow inside the circular cylinder was investigated by Choudhury and Deka [7]. Meksyn application model of steepest descent method was applied, where it was found that Nusselt number and visco-elastic absolute value reduced the shearing stress. Hayat et al. [8] discussed the heat absorption and heterogeneous reactions due to a rotating disk for the second-grade fluid. Appropriate initial guesses were made to assure the solution convergence. Computed results depicted that visco-elastic parameter and Schmidt number were the increasing functions of concentration profile. Shear stress and velocity profile were evaluated by using the fractional derivative approach in Raza et al. [9] model. The hybrid technique involves semi-analytical fractional-order solutions condensed to the ordinary form. Non-Fourier heat flux and thermal conductivity for temperature-dependent fluid were numerically investigated by Hayat et al. [10]. HAM solution showed that the velocity profile accelerated with visco-elastic and curvature parameter. Moreover, temperature decayed with increasing thermal stratification and Prandtl number. Blood and fluid flow problems without singularity in the fractional domain were analytically studied by Uddin et al. [11], [12], [13]\& [14]. Temperature distribution for solid oxide fuel cell studied 
by Guk et al. [15] was subjected to various flow parameters impact. Open circuit voltage conditions and direct hydrogen oxidation was the most significant contributor for the average increment in the cell's temperature. Zhang et al. [16] experimentally investigated lateral smoke extraction. Thermal behaviour across longitudinal ventilation was also studied. Experimental results displayed that the smoke temperature reduced exponentially along with the tunnel ceiling. Curved sidewall effect on flame characteristic and ceiling was experimentally studied by Liang et al. [17]. Inside the tunnel ceiling temperature distribution was found asymmetrical near the fire source region. Shojaei et al. [18] analytically examined the Soret and Dufour effects along stretching cylinder. Both were in negative correlation with heat and mass transfer rate. Xu et al. [19] used a fuel cell device for the direct conversion of chemical energy into electricity. Two-dimensional control orient model of the differential equation was established for the fuel cell device. Thermoelectric characteristics were reflected by the simulated results and especially the temperature distribution across the device.

In this paper heat transfer effect due to natural convection in a vertically oscillating cylinder is focused upon. Previously, analytic solutions were expressed in terms of generalized functions, which were inadequate for simulations. Whereas, in the present research, partial differential equations have been made free from the singular kernel, which makes it more suitable for numerical simulations. Computer software Mathematica was used for simulations. Discussion and graphical illustrations have been made in the end.

\section{Mathematical Model}

Present taking the unusual Boussinesq approximation, the unsteady MHD second grade thermal fluid flow is governed by the following set of partial differential equations

$$
\begin{gathered}
\frac{\partial}{\partial t} u(r, t)=\left(v+\frac{\alpha_{3}}{\rho} \frac{\partial}{\partial t}\right)\left(\frac{\partial^{2} u(r, t)}{\partial r^{2}}+\frac{1}{r} \frac{\partial u(r, t)}{\partial r}\right)-\frac{\sigma B_{0}{ }^{2} u(r, t)}{\rho} \\
+g \beta_{T}\left(T(r, t)-T_{\infty}\right) ; t>0, \\
\frac{\partial}{\partial t} T(r, t)=\frac{k}{\rho C_{p}}\left(\frac{\partial^{2} T(r, t)}{\partial r^{2}}+\frac{1}{r} \frac{\partial T(r, t)}{\partial r}\right) ; t>0, r \in\left[0, R_{0}\right] .
\end{gathered}
$$

The Initial and boundary conditions are

$$
\begin{gathered}
u(r, 0)=0, T(r, 0)=T_{\infty} ; r \in\left[0, R_{0}\right], \\
u\left(R_{0}, t\right)=V_{0} U(t) \exp (i \omega t), T\left(R_{0}, t\right)=T_{w} ; t>0 .
\end{gathered}
$$

Where in Eq.(1) \& (2) $u(r, t)$ is the fluid velocity, $T(r, t)$ is fluid temperature, $v$ is the fluid kinematic viscosity, $\alpha_{3}$ is the second grade fluid parameter, $\rho$ is the fluid density, $\beta_{T}$ is the fluid volumetric coefficient of thermal expansion, $g$ is the gravitational acceleration, $C_{p}$ is the fluid heat capacity at constant pressure and $k$ is the fluid thermal conductivity.

\subsection{Dimensionless Time Fractional Model}

A Governing momentum and energy equations are non-dimensionalised by introducing dimensionless variables. After dropping * notations we have

$$
\begin{aligned}
& \frac{\partial}{\partial t} u(r, t)=\left(1+\alpha_{4} \frac{\partial}{\partial t}\right)\left(\frac{\partial^{2} u(r, t)}{\partial r^{2}}+\frac{1}{r} \frac{\partial u(r, t)}{\partial r}\right)-H a^{2} u(r, t) \\
& +\operatorname{Gr} T(r, t) ; t>0, \\
& \frac{\partial}{\partial t} T(r, t)=\frac{1}{\operatorname{Pr}}\left(\frac{\partial^{2} T(r, t)}{\partial r^{2}}+\frac{1}{r} \frac{\partial T(r, t)}{\partial r}\right) ; t>0, r \in[0,1], \\
& u(r, 0)=0, T(r, 0)=0 ; \quad r \in[0,1], \\
& u(1, t)=U(t) \exp (i \omega t), T(1, t)=1 ; t>0 .
\end{aligned}
$$

Replacing the classical time partial derivative with the Caputo-Fabrizio time fractional derivative of non-integer order $\alpha \in[0,1]$ in eqs (5) and (6) respectively, one obtains:

$$
\begin{gathered}
D_{t}^{(\alpha)} u(r, t)=\left(1+\alpha_{4} D_{t}^{(\alpha)}\right)\left(\frac{\partial^{2} u(r, t)}{\partial r^{2}}+\frac{1}{r} \frac{\partial u(r, t)}{\partial r}\right)-\operatorname{Hau}(r, t) \\
+G r T(r, t) ; t>0, \\
D_{t}^{(\alpha)} T(r, t)=\frac{1}{\operatorname{Pr}}\left(\frac{\partial^{2} T(r, t)}{\partial r^{2}}+\frac{1}{r} \frac{\partial T(r, t)}{\partial r}\right) ; t>0, r \in[0,1],
\end{gathered}
$$

\subsubsection{Temperature Field}

Temperature of second grade fluid can be obtained by taking Laplace and finite Hankel transforms of the energy equation in Eq. (10) along with initial and boundary conditions in Eq. $(7)_{2} \&(8)_{2}$, and we obtain the final analytical form of the temperature profile in the local as well as fractional model $0<\alpha \leq 1$,

$$
T(r, t)=1+2 \sum_{n=1}^{\infty} \frac{r_{n} J_{0}\left(r r_{n}\right)}{J_{1}\left(r_{n}\right) b_{1}}\left\{1-\alpha-\frac{\alpha}{b 2}\right\} \times e^{-b_{2} t}, \text { for } 0<\alpha \leq 1 .
$$

Where $b_{1}=\operatorname{Pr}+r_{n}^{2}-r_{n}^{2} \alpha, b_{2}=r_{n}^{2} \alpha / b_{1} J_{0}$ and $J_{1}$ are the bessel functions of zero and first order with first kind and $r_{n}$, $\mathrm{n}=1,2,3, \ldots$ are the positive roots of $J_{0}(x)=0$.

\subsubsection{Velocity Field}

The Velocity profile of second grade fluid under the action of external applied magnetic field and heat transfer in the fractional model can be obtained by Eq. (9) along with initial and boundary conditions stated in Eq. (7) \& (8) 1 . After taking Laplace and finite Hankel transforms on these equations we have, 


$$
\begin{aligned}
& u(r, t)=e^{i \omega t}+2 \sum_{n=1}^{\infty} \frac{r_{n} J\left(r r_{n}\right)}{f_{2} J_{1}\left(r_{n}\right)}\left[f_{4} \frac{f_{11}}{e^{f_{3} t}}-\alpha e^{i \omega t}+f_{4} f_{10} e^{i \omega t}\right. \\
& +\alpha_{4} e^{i \omega t}-\alpha_{4} f_{3}\left(f_{10} e^{i \omega t}+f_{11} e^{-f_{3} t}\right)+G r\left\{\frac { 1 - \alpha } { b _ { 1 } } \left(\frac{1-\alpha}{e^{b_{2} t}}\right.\right. \\
& \left.-\frac{\alpha\left(e^{-b_{2} t}-1\right)}{b_{2}}\right)+\frac{f_{4}}{b_{1}}(1-\alpha)\left(f_{5} e^{-f_{3} t}+f_{6} e^{-b_{2} t}\right)+\frac{f_{4} \alpha}{b_{1}} \\
& \left.\left.\times\left(f_{7}+\frac{f_{8}}{e^{b_{2} t}}+\frac{f_{9}}{e^{f_{3} t}}\right)\right\}\right], \text { for } 0<\alpha \leq 1 .
\end{aligned}
$$

Where

$$
\begin{gathered}
f_{1}=r_{n}{ }^{2}+\mathrm{Ha}^{2}, \\
f_{2}=1+f_{1}-f_{1} \alpha+\alpha_{4} r_{n}{ }^{2}, \\
f_{3}=\frac{f_{1} \alpha}{f_{2}}, \\
f_{4}=\alpha-f_{3}(1-\alpha), \\
f_{5}=\frac{1}{b_{2}-f_{3}}, \\
f_{6}=\frac{1}{f_{3}-b_{2}}, \\
f_{7}=\frac{1}{f_{3} b_{2}}, \\
f_{8}=\frac{-1}{b_{2}\left(f_{3}-b_{2}\right)}, \\
f_{9}=\frac{-1}{f_{3}\left(b_{2}-f_{3}\right)}, \\
f_{10}=\frac{1}{f_{3}+i \omega},
\end{gathered}
$$

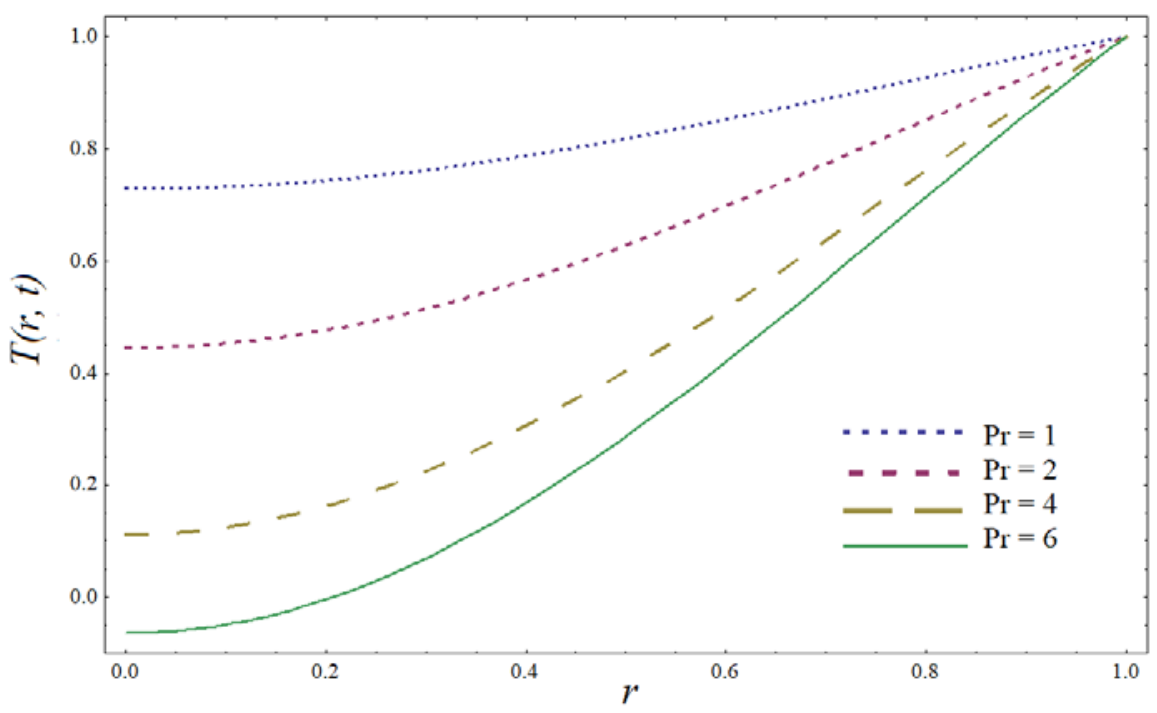

Figure 2. Temperature profile $T(r, t)$ against $r$

$$
f_{11}=\frac{-1}{f_{3}+i \omega}
$$

\subsection{Numerical Results and Discussion}

By using Eq. (11) \& (12), influence of flow parameters like Prandtl number and external magnetic field on temperature and velocity profile is investigated. Fig (1) shows the flow geometry. For computer-based simulations other fixed flow parameters are $\alpha_{4}=0.5$ and 1 (second grade fluid parameter), $\operatorname{Pr}=1,2,4$ and 6 (Prandtl number), $G r=1$ (Grashof number), $\omega=\frac{P i}{4}$ and $\mathrm{Ha}=2,3,4$ and 5 .

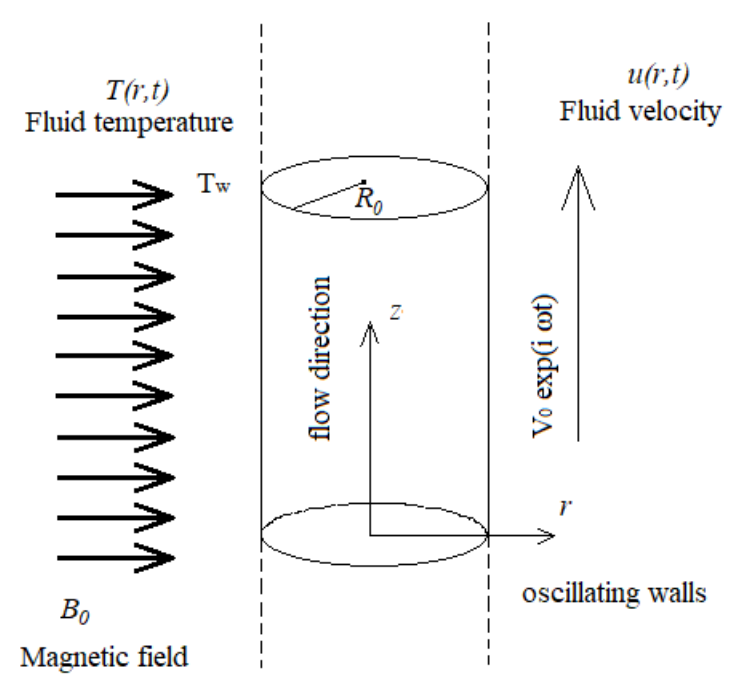

Figure 1. Fluid flow geometry inside oscillating walls 


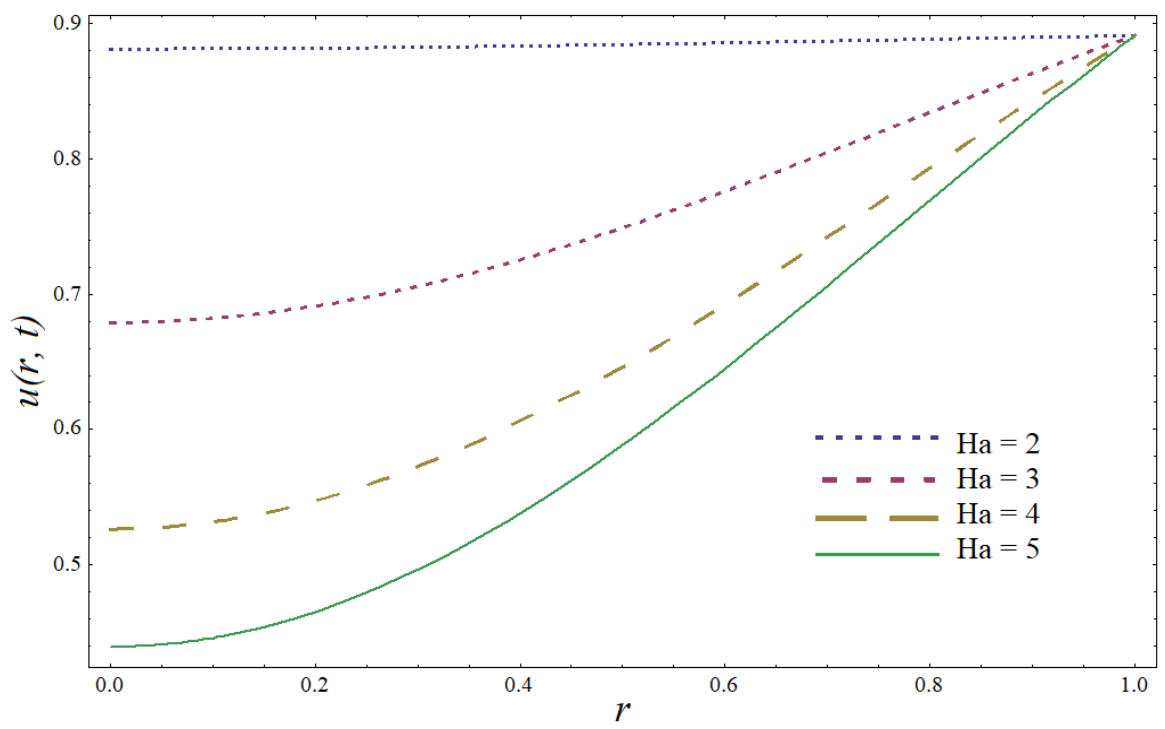

Figure 3. Velocity profile $u(r, t)$ against $r$

In Fig. (2), the effect of Prandtl number on temperature profile for fractional second grade non-Newtonian fluid is shown. It is observed that heat transfer from oscillating cylinder towards the fluid is significant and fluid gets warm for small Pr and begins to cool by increasing values due to thickening of the thermal boundary layer.

Fluid flow was exposed to the external magnetic field at different strengths in Fig. (3), $\mathrm{Ha}=2,3,4$ and 5 against $r$. Fluid velocity decreased with the magnetic field.

\section{Conclusions}

The article concluded by mentioning the following main points that:

1. Thickening of the thermal boundary layer decreases the fluid temperature.

2. Fluid flow can be controlled by applying sufficiently strong magnetic field.

It is expected that the present study can inspire other researchers as well. The study can be extended to the stretching flow models in the food process, blood flow, paper production and polymeric solutions.

\section{Acknowledgements}

I would like to acknowledge the financial aid received under Tier 1/H070 grant from Universiti Tun Hussein Onn Malaysia.

\section{REFERENCES}

[1] A. S. Nehad, K. Ilyas. Heat transfer analysis in a second grade fluid over and oscillating vertical plate using fractional Caputo-Fabrizio derivatives, Eur. Phys. J. C, Vol.76, 1-12, 2016.

[2] D. Kalidas, P. S. Ram, S. Amit, Heat and mass transfer of a second grade magnetohydrodynamic fluid over a convectively heated stretching sheet, Journal of Computational Design and Engineering, Vol.3, 330-336, 2016.

[3] R. Abdul, A. Sallahuddin, N. Sohail, I. Saleem, Stagnation point flow of Eyring Powell fluid in a vertical cylinder with heat transfer, Journal of Power Technologies, Vol.96, No.1, 57-62, 2016.

[4] V. Ramachandrprasad, R. Bhuvanavijaya, B. Mallikarjuna, Natural convection on heat transfer flow of non-newtonian second grade fluid over horizontal circular cylinder with thermal radiation, Journal of Naval Architecture and Marine Engineering, Vol.13, 63-78, 2016.

[5] F. I. Alao, A. I. Fagbade, B. O. Falodun, Effects of thermal radiation, Soret and Dufour on an unsteady heat and mass transfer flow of a chemically reacting fluid past a semi-infinite vertical plate with viscous dissipation, Journal of the Nigerian Mathematical Society, Vol.35, 142-158, 2016.

[6] G. Taza, G. Fazle, S. Islam, R. A. Shah, I. Khan, N. Saleem, S. Sharidan, Unsteady thin film flow of a fourth grade fluid over a vertical moving and oscillating belt, Propulsion and Power Research, Vol.5, No.3, 223-235, 2016.

[7] C. Rita, D. Bibhash, MHD Visco-elastic fluid flow and heat transfer around a circular cylinder, Wseas Transactions On Fluid Mechanics Vol.12, 98-107, 2017.

[8] H. Tasawar, Q. Sumaira, A. Ahmed, A. Bashir, Significant consequences of heat generation/absorption and Homogeneous-heterogeneous reactions in second grade fluid due to rotating disk, Results in Physics 8, 223-230, 2018.

[9] R. Nauman, M. Abdullah, R. B. Asma, U. A. Aziz, U. H. Ehsan, Flow of a second grade fluid with fractional 
derivatives due to a quadratic time dependent shear stress. Alexandria Engineering Journal, Vol.57, 1963-1969, 2018.

[10] T. Hayat, A. Salman, A. I. Khan, A. Alsaedi, M. Waqas, Investigation of second grade fluid through temperature dependent thermal conductivity and non-Fourier heat flux. Results in Physics, Vol.9, 871-878, 2018.

[11] S. Uddin, M. Mohamad, K. Khalid, M. Abdulhameed, M. S. Rusiman, N. Che-Him, R. Roslan, Blood flow problem in the presence of magnetic particles through a circular cylinder using Caputo-Fabrizio fractional derivative, IOP Conference Series: Journal of Physics: Conf. Series, Vol.995, 1-9, 2018.

[12] S. Uddin, M. Mohamad, M. A. H. Mohamad, S. Sufahani, M. Kamardan, O. U. Mehmood, F. Wahid \& R. Roslan, Caputo-Fabrizio time fractional derivative applied to visco elastic MHD fluid flow in the porous medium, International Journal of Engineering and Technology, Vol.7, No.4.30, 533-537, 2018.

[13] S. Uddin, M. Mohamad, S. Sufahani, M. Kamardan, O. U. Mehmood, F. Wahid \& R. Roslan, Application of Caputo-Fabrizio fractional order derivative(NFDt) in simulating the magnetohydrodynamic (MHD) flow of the third grade non-Newtonian fluid in the porous artery, International Journal of Engineering and Technology, Vol.7, No.4.30, 527-532, 2018.

[14] S. Uddin, M. Mohamad, M. R. Gorji, R. Roslan, I. M. Alarifi, Fractional electro-magneto transport of blood modeled with magnetic particles in cylindrical tube without singular kernel, Microsystem Technologies, Vol.25, 1-10, 2019.

[15] S. Uddin, M. Mohamad, M. Kamardan, M. A. H. Mohamad, S. Sufahani, R. Roslan, Electromagnetic control of fluid with magnetic particles in the stenosed region, IOP Conference Series: Materials Science and Engineering, Vol.551, No.1, 1-6, 2019.

[16] G. Erdagon, V. Vijay, B. Shumaila, J. Lisa, S. K. Jung, Parameters and their impacts on the temperature distribution and thermal gradient of solid oxide fuel cell, Applied Energy Vol. 241, 164-173, 2019.

[17] Z. Xin, X. Zhisheng, N. Tianxiao, P. Jinzhi, Z. Jiawei, Investigation on smoke temperature distribution in a double-deck tunnel fire with longitudinal ventilation and lateral smoke extraction, Case Studies in Thermal Engineering, Vol.13, 1-9, 2019.

[18] H. L. Zhen, Q. Z. Guo, N. L. Hao, Z. Xiang, Flame characteristic and ceiling temperature distribution under the effect of curved sidewall, Case Studies in Thermal Engineering 14, 1-8, 2019.

[19] S. Ahmadreza, A. A. Jafarian, S. A. Saedi, K. Hosseinzadeh, D. D. Ganji, Hydrothermal analysis of Non-Newtonian second grade fluid flow on radiative stretching cylinder with Soret and Dufour effects, Case Studies in Thermal Engineering, Vol.13, 1-14, 2019.

[20] W. X. Yuan, L. W. Xiao, Y. Hang, X. Tao, Q. Z. Dong, H. J. Jian, H. D. Zhong, W. F. Xiao, X. Li, Modeling and simulation of temperature distribution for planar cross-flow solid oxide fuel cell, Energy Procedia, Vol.158, 1585-1590, 2019.

[21] C. Li, D. Qian, Y. Q. Chen, On Riemann-Liouville and
Caputo Derivatives, Discrete dynamics in nature and society, 1-16, 2011.

[22] M. Caputo, M. Fabrizio, A new Definition of Fractional Derivative without Singular Kernel. Progress in Fractional Differentiation and Applications, Vol.1, No.1, 73-85, 2015. 